\title{
Correlation and modelling of the penetration and softening point tests of bitumen binders with a variety of physical and chemical properties
}

\author{
Marvy Girgis (D), Jérémie Barbier*, Alain Quignard, Isabelle Merdrignac, and João Marques \\ IFP Energies nouvelles, Rond-point de l'échangeur de Solaize, BP 3, 69360 Solaize, France
}

Received: 21 January 2021 / Accepted: 29 July 2021

\begin{abstract}
Studying bitumen has always posed a challenge to researchers owing to its extreme complexity and unique properties. To classify it commercially and to determine bitumen grade, two standard empirical tests have been adopted within the European standardized bitumen binders system [EN 12591:2009 (2009) Bitumen and bituminous binders - Specifications for paving grade bitumens]: Softening Point (SP) and Penetration (PEN). The relationship between these two tests and the physical or chemical properties of bitumen is not well understood. For the first time, this study represents an attempt to build more understanding of such a relationship through a comprehensive study of the correlation between the two standard tests and many physical and chemical properties of bitumen. A second goal is to propose some predictive models for these two tests and compare their predictive accuracy. Therefore, 13 Straight Run Vacuum Residues (SRVR) samples from different geographical origins were analyzed to measure the following parameters: Dynamic Viscosity (VisDy), Conradson Carbon Residue (CCR), C5 Asphaltenes Content (AspC5), C7 Asphaltenes Content (AspC7), Elemental Analysis (including C, H, O, N, S, Ni, and V content), Simulated Distillation (SD), FourierTransform Infrared Spectroscopy (FT-IR), and proton nuclear magnetic resonance spectroscopy (H-NMR). Results of studying correlations using correlation matrix and Principal Component Analysis (PCA) have emphasized the prominent effect of asphaltenes content on the other properties and the results of SP and PEN. It has also shown the potential importance of the aliphaticity/aromaticity of bitumen. Then, four models were proposed for the prediction of SP and PEN: viscosity, FT-IR, H-NMR, and multi-parameter models. Partial least squares (PLS) regression was used for building all models, except viscosity ones. All SP models, except H-NMR model, exhibited very good accuracy compared to the standard method. On the other hand, PEN was more difficult to predict than SP and only the multi-parameter model of PEN showed relatively good accuracy of prediction.
\end{abstract}

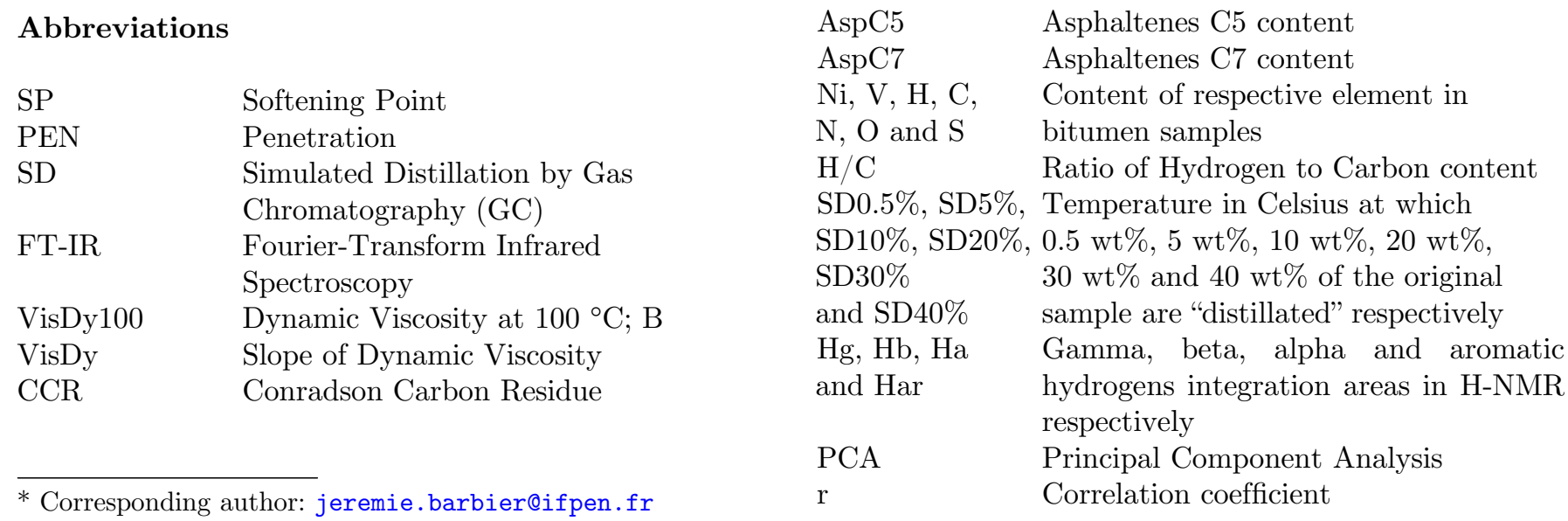




\section{Introduction}

Bitumen (or Asphalt) is the most widespread material used for road surface constructions and pavement [1-3]. The main source of bitumen production is the Straight Run (SR) production of the heaviest fraction (i.e. the Vacuum Residue or VR) of specific crude oils through the fractional distillation process: SRVR. Nowadays, more than $85 \%$ of the produced bitumen is used in road constructions due to many advantages like cost-effective, environmentally friendly, and very good durability allowing better energy balance for motor vehicles. Moreover, pavement roads can be easily repaired and bitumen is also recyclable [4]. Many other uses of bitumen exist based on its waterproofing and adhesive properties [3]. In this decade, there is a large diversity of bitumen's sources and some unconventional bitumen binders started to appear in the market that are not produced by the usual SR process. With the evolution of the Sulphur restrictions (less than $0.5 \mathrm{wt} \%$ ) in bunker fuels since January 2020, the market of high sulfur residual fuel oils has decreased with a possible shift to a new market as bitumen binders. But since only about $10 \%$ of the world crude oils are used for bitumen production, the question is: What about "non-bitumen" resources as bitumen binders?

Chemically, bitumen is a complex mixture of hundreds of thousands of compounds that are mostly hydrocarbons with a small amount of heterocyclic species and functional groups containing sulphur, nitrogen, and oxygen atoms in addition to traces of heavy metals. Full chemical analysis of bitumen is practically impossible, even with modern and high performances analytical techniques, due to its high complexity. In addition, the chemical composition of the produced bitumen may differ greatly depending on several factors such as crude oil origin, type, and operating conditions of the production process $[3,5,6]$. Different attempts to characterize bitumen have been based on bulk properties such as solubility in different solvents, molecular weight, or broad chemical type. Over the past and present centuries, many efforts have been made in order to characterize the physical and chemical properties of bitumen using different analytical techniques [6-9]. For example, liquid chromatographic has been widely used to separate bitumen into different fractions $[6,7]$. The most common way is to separate bitumen into four fractions called SARA fractions: saturates, aromatics, resins, and asphaltenes, which exhibit an increase in aromaticity, polarity, viscosity, and average molecular weight as we move from saturates to asphaltenes $[2,3,5]$. Other analytical techniques like Fourier Transform Infrared (FT-IR) spectroscopy, Nuclear Magnetic Resonance (NMR), or mass spectroscopy can help to provide relevant information about the average chemical composition of bitumen [6, 10-13]. Besides, techniques like Gel-Permeation Chromatography (GPC), also called Size Exclusion Chromatography or SEC, Atomic Force Microscopy (AFM), Thermogravimetric Analysis (TGA), Differential Scanning Calorimeter (DSC) and Simulated Distillation (SD) by High-Temperature Gas Chromatography (HTGC) and low-field NMR can give more insights into the molecular dynamics that affect physical properties of bitumen $[6,9,10,14,15]$.
For a suitable determination of bitumen performance in different environments, different systems of classifications have been adopted based on empirical tests (like penetration grade classes, European classification EN 12591 [1]) or fundamental tests based on rheology (like Superpave specifications) [3, 16]. According to the European Standard EN 12591 [1], the penetration grade of bitumen is determined by two main characterization tests: Penetration (PEN) and Softening Point (SP) measures [17, 18]. The penetration and softening point of bitumen are used to characterize the consistency and stiffness of bitumen at ambient and high service temperature respectively, which are undoubtedly affected by the physical and chemical properties of bitumen $[3,16]$. The interpretation of these two standard tests is difficult and cannot be directly linked to the temperature susceptibility or the viscoelastic properties of bitumen [16]. However, the penetration grade classes system is still widely used in European countries for bitumen classification.

In consequence, researchers have put a lot of effort to correlate and possibly model the penetration and softening point test of bitumen with its different physical or chemical properties by using different analytical techniques. It was found that an increase of viscosity leads to a SP increase and a PEN decrease. Some models have been proposed to predict SP or PEN from viscosity [19, 20]. A proven relationship between SARA fractions and SP or PEN has been demonstrated by many researchers [14, 21, 22]. For instance, increasing asphaltenes content has a similar effect to viscosity on PEN and SP. Weigel and Stephan [11] proposed prediction models of PEN and SP based on the contents and the molecular weights of SARA fractions. A more recent trend is the use of spectroscopic techniques to model SP and PEN, like Fourier Transform Infrared Spectroscopy (FT-IR) and Nuclear Magnetic Resonance (NMR). Jaimes et al. proposed some models for SP and PEN predictions based on Low Field NMR (LF-NMR) and H-NMR [10]. However, most of the proposed predictive models of SP or PEN were built by using samples with narrow ranges of SP and PEN, and the predicted results are relatively unreliable compared to the standard methods. To the best of our knowledge, no comparison has been made between the different analytical approaches used for SP and PEN predictions. Since every author has used this own set of samples to develop the models, and on a quite limited prediction window, the performance of each approach has not been yet compared using the same set of bitumen samples.

Therefore, the objective of this study is to investigate the correlation between SP, PEN, and a variety of physical and chemical properties and to compare the predictive performance of the following four proposed models for SP and PEN predictions: dynamic viscosity, FT-IR, H-NMR, and multiparameter models.

\section{Materials and methodology}

\subsection{Bitumen samples}

The study has involved 13 samples of bitumen, which were obtained from different crude oil straight run distillation. 
Table 1. Characteristics of the studied bitumen samples.

\begin{tabular}{llccc}
\hline Sample name & Geographical origin of crude oil & Density & Penetration $(\mathrm{dmm})$ & Softening point $\left({ }^{\circ} \mathrm{C}\right)$ \\
\hline B1 & Canada & 1.047 & 16 & 61 \\
B2 & Venezuela & 1.039 & 18 & 77 \\
B3 & Saudi Arabia & 1.063 & 22 & 61 \\
B4 & China & 1.037 & 26 & 72 \\
B5 & Saudi Arabia & 0.955 & 63 & 50 \\
B6 & Saudi Arabia & 1.024 & 83 & 48 \\
B7 & Rep. Congo & 0.979 & 123 & 44 \\
B8 & Russia & 1.014 & 134 & 42 \\
B9 & China & 0.994 & 223 & 39 \\
B10 & Saudi Arabia & 1.021 & 283 & 34 \\
B11 & North Europe & 1.011 & 286 & 33 \\
B12 & Russia & 1.000 & 291 & 38 \\
B13 & Russia & 1.006 & 318 & 32 \\
\hline
\end{tabular}

The list of the samples is presented in Table 1. The samples are distillation residues, which differ from each other in terms of the geochemical and geographical origin of the crude oils used for distillation, with a wide $\mathrm{SP} / \mathrm{PEN}$ range. Due to the important diversity of origin, the sample characteristics such as the density, penetration, and softening point are relatively diversified. This variability indicates that the studied samples have different chemical compositions.

\subsection{Analytical characterizations}

Density was measured according to the NF EN ISO 12185 method. Softening Point (SP) was measured according to the NF EN 1427 method. Penetration (PEN) was measured according to the NF EN 1426 method. Dynamic viscosity was measured according to the ASTM D3236 method. Regarding dynamic viscosity, it was measured at $100{ }^{\circ} \mathrm{C}$ (VisDy100) and $130{ }^{\circ} \mathrm{C}$ to calculate the slope of viscosity against temperature $(B)$ according to equation (1):

$$
\boldsymbol{B}=\frac{\ln \left[\frac{\ln (\mathrm{VisD} 100+\mathbf{0 . 7})}{\ln (\mathrm{VisDy} 130+\mathbf{0 . 7})}\right]}{\ln \left(\frac{130}{100}\right)} .
$$

Conradson Carbon Residue (CCR) was measured according to the microCCR method NF EN ISO 10370 method. Content of asphaltenes precipitated in n-pentane and n-heptane (AspC5, AspC7, respectively) was calculated according to the procedure of the NFT60-115 method. Elementary composition $(\mathrm{C}, \mathrm{H}, \mathrm{O}, \mathrm{N}, \mathrm{S})$ was determined by combustion methods. $\mathrm{Ni}$ and $\mathrm{V}$ contents were measured according to the ASTM D7260 method. Simulated distillation was performed according to the ASTM D7269 method. The temperatures at which a weight loss of $0.5,5 \%, 10 \%$, $20 \%, 30 \%$, and $40 \%$ of the original sample were measured.

For Fourier-Transform Infrared (FT-IR) spectroscopy measurement, samples were heated at a temperature of $130{ }^{\circ} \mathrm{C}$, and then they were applied directly and uniformly on an ATR Type IIIa diamond brazed crystal in a tungsten carbide piece to ensure full surface contact and left to cool down until they reach room temperature before performing the measurement. This method avoids solvent addition to the sample. The equipment used is Nicolet ${ }^{\circledR}$ Nexus with a resolution of $4 \mathrm{~cm}^{-1}$ and 64 scans per sample. Three replicate measurements were done for each sample and the average spectrum was used. Due to the presence of atmospheric $\mathrm{CO}_{2}$ in some spectra and based on peak intensity, only the relevant wave number ranges between 3600 and $2500 \mathrm{~cm}^{-1}$ and 1800 and $690 \mathrm{~cm}^{-1}$ were considered.

For H-NMR spectrometry, samples solubilized in $\mathrm{CDCl}_{3}$ were characterized with Bruker $600 \mathrm{MHz}$ NMR Avance III. For each sample, 256 scans were performed. A quantitative mode was utilized based on the calculation of relaxation time and determination of the proper acquisition time in NMR. To ensure the quantitative accuracy of the H-NMR, it is of utmost importance to achieve the full relaxation of the nuclei between pulses. The relaxation decay (d1) should be at least five times the $\mathrm{T} 1$ relaxation of the slowest relaxing signal of interest. The T1 was found to be less than $1 \mathrm{~s}$ so $5 \mathrm{~s}$ was chosen as the value for $\mathrm{d} 1$. In addition, the TMS shift was calculated for the developed method. Spectra treatment was done using Top Spin 3.6.2 software. Phase correction was applied to all spectra and areas in the spectrum were integrated according to the chemical shift range $0.5-1.0 \mathrm{ppm}$ related to methyl protons on a $\gamma$ or more carbon $(\mathrm{Hg}) ; 1.0-1.9 \mathrm{ppm}$ related to protons attached to a saturated $\beta$ or more carbon $(\mathrm{Hb}) ; 1.9-4.5 \mathrm{ppm}$ related to protons attached to saturated $\alpha$ carbon (Ha) and $6.3-9.3 \mathrm{ppm}$ related to aromatic protons (Har).

According to the different performed analyses, Table 2 shows how the samples of this study exhibit large diversity and variability in properties, in terms of range, mean and standard deviation of each parameter.

\subsection{Modelling}

The following methodology for modelling has included two steps: the first step is to study the correlation between 
Table 2. Summary of the results of different analytical techniques ${ }^{\text {(a) }}$ used in all bitumen samples analysis (except FTIR), which include range (minimum - maximum), mean and standard deviation (SD). The result shows clearly the diversity of the samples under investigation.

\begin{tabular}{lccccc}
\hline Parameters & Range & Mean \pm SD & Parameter & Range & Mean \pm SD \\
\hline VisDy100 (mPa.s) & $533-54775$ & $10449 \pm 16542$ & SD0.5\% $\left({ }^{\circ} \mathrm{C}\right)$ & $305.8-461$ & $401.9 \pm 47$ \\
B VisDy & $3.27-5.65$ & $3.65 \pm 0.62$ & SD5\% $\left({ }^{\circ} \mathrm{C}\right)$ & $443.5-528.5$ & $494.1 \pm 23.4$ \\
CCR $(\% \mathrm{~m} / \mathrm{m})$ & $14.9-27.6$ & $19.9 \pm 4.2$ & SD10\% $\left({ }^{\circ} \mathrm{C}\right)$ & $476.7-552.2$ & $520.6 \pm 19.7$ \\
AspC5 $(\% \mathrm{~m} / \mathrm{m})$ & $8.4-31.9$ & $18.6 \pm 9.4$ & $\mathrm{SD} 20 \%\left({ }^{\circ} \mathrm{C}\right)$ & $522.6-585.3$ & $554.3 \pm 16.6$ \\
$\mathrm{AspC}(\% \mathrm{~m} / \mathrm{m})$ & $2.1-23.5$ & $10.1 \pm 7.1$ & $\mathrm{SD} 30 \%\left({ }^{\circ} \mathrm{C}\right)$ & $561.2-613.3$ & $582.7 \pm 14.3$ \\
$\mathrm{Ni}(\mathrm{mg} / \mathrm{Kg})$ & $28-115$ & $64 \pm 23$ & $\mathrm{SD} 40 \%\left({ }^{\circ} \mathrm{C}\right)$ & $587.6-640.9$ & $610.4 \pm 14.0$ \\
$\mathrm{~V}(\mathrm{mg} / \mathrm{Kg})$ & $9-450$ & $195 \pm 132$ & $\mathrm{Hg}(\%)$ & $12.61-16.95$ & $15.08 \pm 1.14$ \\
$\mathrm{C}(\% \mathrm{~m} / \mathrm{m})$ & $82.7-87.5$ & $84.9 \pm 1.4$ & $\mathrm{Hb}(\%)$ & $52.49-68.84$ & $63.44 \pm 3.91$ \\
$\mathrm{H}(\% \mathrm{~m} / \mathrm{m})$ & $9.6-11.2$ & $10.4 \pm 0.5$ & $\mathrm{Ha}(\%)$ & $9.54-17.54$ & $14.34 \pm 2.14$ \\
$\mathrm{H} / \mathrm{C}$ & $0.11-0.13$ & $0.12 \pm 0.00$ & $\mathrm{Har}(\%)$ & $4.68-16.89$ & $7.15 \pm 3.00$ \\
$\mathrm{~N}(\mathrm{mg} / \mathrm{Kg})$ & $4011-7545$ & $5841 \pm 1184$ & $\mathrm{SP}\left({ }^{\circ} \mathrm{C}\right)$ & $31.8-76.6$ & $48.5 \pm 14.8$ \\
$\mathrm{O}(\% \mathrm{~m} / \mathrm{m})$ & $0.22-0.97$ & $0.52 \pm 0.19$ & $\mathrm{PEN}(\mathrm{dmm})$ & $16-318$ & $145 \pm 119$ \\
$\mathrm{~S}(\% \mathrm{~m} / \mathrm{m})$ & $0.4-5.9$ & $3.5 \pm 1.7$ & & & \\
\hline
\end{tabular}

SP, PEN, and the different measured properties. This first step is essential to select the main relevant parameters for the second step: modelling. Correlation matrix using data analysis tool pack in Excel $^{\circledR}$, as well as loading plot from PCA, were used to sort the data. For biplots of PCA, R Studio $^{\circledR}$ was used together with ggplot2 package. PCA alone was used to detect possible outliers or atypical samples.

For the second step, modelling was done using Partial Least Square (PLS) regression. PLS is essentially Linear Regression (LR) technique based on PCA (Principal Component Analysis), which is a data reduction that converts a large number of variables that are correlated to each other to fewer non-correlated components. Coefficient of determination $\left(R^{2}\right)$, Root Mean Square Error (RMSE), and Bias are used as parameters for determining the quality of the model. Calculations were done using SOLO $^{\circledR}$ PLS tool pack. For each model evaluation, cross-validation using Leave One Out (LOO) method was used.

\section{Results and discussion}

\subsection{Correlation between different parameters and SP or PEN}

The correlation matrix was used to detect the parameters that are significantly correlated with SP or PEN (Tab. 3). The level of significance of the correlation coefficients $(r)$ in this matrix was chosen to be outside the range -0.3 to 0.3 (according to the guideline of The Political Science Department at Quinnipiac University) [23]. In other words, any two parameters that have a correlation coefficient with a value below -0.3 or above 0.3 are considered significantly correlated. Parameters with $r$ value outside the range -0.4 to 0.4 are considered moderately correlated and those with $r$ values above the range -0.7 to 0.7 should have a strong correlation. The sign of the correlation coefficient defines the direction of the correlation; it is either a positive correlation or a negative one. SP showed a significant correlation with all parameters except the following: B VisDy, N, O, SD0.5\%, SD20\%, SD30\%, and Hg (Tab. 3). All parameters that are significantly correlated with SP displayed the same behavior with PEN but opposite in direction (except SD5\% and SD10\%, which are also not significantly correlated to $\mathrm{PEN})$. This can be explained by the strong negative correlation between SP and PEN $(r=-0.89)$. The strongest correlations were recorded between either SP or PEN and CCR, AspC5, AspC7, H, and $\mathrm{H} / \mathrm{C}$ ratio. VisDy100 and V were also strongly and positively correlated with SP.

Principal Component Analysis (PCA) was also applied to further assess the correlation between different parameters and SP or PEN. The first seven PCs explain more than $95 \%$ of the variance of the data. The first 3 PCs account for $78.7 \%$ of the total explained variance of the 25 parameters included in this study. Figure 1 displays the biplot of the first Principal Component (PC1) and the second Principal Component (PC2). The biplot of the PCA provides an excellent representation of the relationship between the scores (samples) and the loadings (parameters). Besides detecting correlations between different parameters, this method of visualization is extremely useful to see if there are trends, groups of samples, and to detect atypical, or outlier samples [24]. Samples outside the circle (95\% confidence interval) are potential outliers or atypical samples. In this case, only one outlier was detected (sample B4, which comes from PR China). No distinct clusters of samples appear in this biplot based on their PEN values. However, it has been noticed that PC1 separates samples into two groups: one group with relatively high PEN (> $100 \mathrm{dmm}$ : positive scores values on PC1) and another one with relatively low PEN ( $<100$ dmm: negative scores on PC1). Regarding the loadings, if the parameter gets closer to the circle in Figure 1, it means that this parameter is more well represented in these dimensions $(\mathrm{PC} 1 / \mathrm{PC} 2)$ and has a high correlation with either $\mathrm{PC} 1$ or $\mathrm{PC} 2$ (which depends on its 
Table 3. Correlation coefficients $(r)^{\mathrm{a}}$ between different parameters, SP, and PEN.

\begin{tabular}{|c|c|c|c|c|c|c|c|c|c|c|c|c|c|c|c|c|c|c|c|c|c|c|c|c|c|}
\hline Parameters & $\begin{array}{c}\text { VisDy } \\
100\end{array}$ & $\begin{array}{c}\text { B } \\
\text { VisDy }\end{array}$ & CCR & $\begin{array}{c}\text { Asp } \\
\text { C5 }\end{array}$ & $\begin{array}{c}\text { Asp } \\
\text { C7 }\end{array}$ & $\mathbf{N i}$ & $\mathbf{v}$ & C & H & $\mathrm{H} / \mathrm{C}$ & $\mathbf{N}$ & $\mathbf{o}$ & $\mathbf{s}$ & $\begin{array}{c}\text { SD } \\
0.5 \%\end{array}$ & $\begin{array}{l}\text { SD } \\
\mathbf{5 \%} \%\end{array}$ & $\begin{array}{c}\text { SD } \\
10 \%\end{array}$ & $\begin{array}{c}\text { SD } \\
20 \%\end{array}$ & $\begin{array}{c}\text { SD } \\
30 \%\end{array}$ & $\begin{array}{c}\text { SD } \\
40 \%\end{array}$ & Hg & $\mathbf{H b}$ & $\mathrm{Ha}$ & Har & SP & PEN \\
\hline B VisDy & -0.04 & 1.00 & & & & & & & & & & & & & & & & & & & & & & & \\
\hline CCR & 0.65 & 0.21 & 1.00 & & & & & & & & & & & & & & & & & & & & & & \\
\hline AspC5 & 0.77 & 0.13 & 0.91 & 1.00 & & & & & & & & & & & & & & & & & & & & & \\
\hline AspC7 & 0.83 & 0.24 & 0.91 & 0.95 & 1.00 & & & & & & & & & & & & & & & & & & & & \\
\hline $\mathrm{Ni}$ & 0.35 & -0.14 & -0.03 & 0.21 & 0.18 & 1.00 & & & & & & & & & & & & & & & & & & & \\
\hline $\mathbf{V}$ & 0.80 & 0.03 & 0.56 & 0.65 & 0.77 & 0.43 & 1.00 & & & & & & & & & & & & & & & & & & \\
\hline $\mathrm{H}$ & -0.54 & -0.25 & -0.93 & -0.84 & -0.84 & 0.00 & \begin{tabular}{r|r}
-0.59 \\
\end{tabular} & 0.78 & 1.00 & & & & & & & & & & & & & & & & \\
\hline $\mathrm{H} / \mathrm{C}$ & -0.62 & -0.19 & -0.94 & -0.84 & -0.87 & 年 & \begin{tabular}{r|r}
-0.59 \\
\end{tabular} & 0.57 & $\begin{array}{l:l}0.96 \\
\end{array}$ & 1.00 & & & & & & & & & & & & & & & \\
\hline $\mathbf{N}$ & 0.10 & -0.33 & -0.49 & -0.32 & -0.26 & 0.57 & 0.05 & \begin{tabular}{l|l}
0.71 \\
\end{tabular} & 0.55 & 0.40 & 1.00 & & & & & & & & & & & & & & \\
\hline O & 0.15 & 0.03 & -0.18 & -0.03 & 0.05 & 0.55 & 0.34 & 0.03 & 0.03 & 0.03 & 0.58 & 1.00 & & & & & & & & & & & & & \\
\hline $\mathbf{S}$ & 0.27 & 0.28 & 0.74 & 0.66 & 0.59 & -0.01 & \begin{tabular}{l|l|}
0.43 \\
\end{tabular} & \begin{tabular}{l|l}
-0.98 \\
\end{tabular} & -0.87 & -0.70 & -0.75 & -0.12 & 1.00 & & & & & & & & & & & & \\
\hline SD0.5\% & -0.16 & 0.19 & -0.08 & -0.35 & -0.27 & -0.14 & $\begin{array}{l}-0.25 \\
\end{array}$ & 0.07 & $\begin{array}{l:l}0.09 \\
\end{array}$ & 0.08 & -0.11 & -0.41 & -0.05 & 1.00 & & & & & & & & & & & \\
\hline SD40\% & 0.17 & 0.21 & \begin{tabular}{l|l}
0.46 \\
\end{tabular} & \begin{tabular}{l|l|}
0.32 \\
\end{tabular} & 0.24 & 0.24 & -0.06 & -0.28 & -0.34 & \begin{tabular}{|c|}
-0.33 \\
\end{tabular} & -0.17 & -0.36 & \begin{tabular}{l|l|l}
0.32 \\
\end{tabular} & 0.54 & 0.57 & \begin{tabular}{l|l}
0.63 \\
\end{tabular} & 0.77 & 0.93 & 1.00 & & & & & & \\
\hline $\mathrm{Hg}$ & 0.04 & -0.25 & -0.39 & -0.14 & -0.15 & 0.39 & -0.07 & 0.48 & 0.49 & 0.42 & 0.58 & 0.44 & -0.57 & -0.30 & -0.42 & -0.42 & -0.42 & -0.40 & -0.31 & 1.00 & & & & & \\
\hline $\mathbf{H b}$ & -0.48 & -0.16 & -0.64 & \begin{tabular}{r|r}
-0.60 \\
\end{tabular} & -0.73 & -0.01 & \begin{tabular}{r|}
-0.65 \\
\end{tabular} & \begin{tabular}{l|l}
0.30 \\
\end{tabular} & 0.70 & 0.78 & 0.10 & -0.36 & \begin{tabular}{r|r}
-0.39 \\
\end{tabular} & 0.34 & 0.62 & 0.64 & 0.57 & 0.42 & 0.18 & 0.05 & 1.00 & & & & \\
\hline $\mathrm{Ha}$ & 0.20 & 0.30 & 0.64 & 0.50 & 0.51 & -0.02 & 0.48 & -0.83 & -0.84 & -0.73 & -0.54 & 0.13 & 0.88 & -0.08 & -0.05 & -0.06 & -0.05 & 0.05 & 0.16 & -0.67 & -0.56 & 1.00 & & & \\
\hline Har & 0.47 & 0.08 & \begin{tabular}{l|l}
0.53 \\
\end{tabular} & 0.48 & 0.65 & -0.12 & 0.54 & 0.03 & -0.50 & -0.66 & 0.03 & 0.21 & 0.09 & -0.27 & -0.61 & -0.63 & -0.55 & -0.42 & -0.23 & 0.02 & -0.92 & 0.27 & 1.00 & & \\
\hline SP & 0.91 & 0.09 & 0.81 & 0.91 & 0.93 & \begin{tabular}{l|l|}
0.43 \\
\end{tabular} & 0.74 & -0.34 & -0.69 & -0.76 & 0.02 & 0.17 & 0.42 & -0.24 & -0.39 & -0.33 & -0.17 & \begin{tabular}{|l|} 
\\
\end{tabular} & 0.37 & 0.03 & -0.58 & 0.33 & 0.52 & 1.00 & \\
\hline PEN & -0.64 & -0.22 & -0.80 & \begin{tabular}{|l|l|}
-0.88 \\
\end{tabular} & -0.82 & -0.41 & $\begin{array}{c}-0.50 \\
\end{array}$ & 0.41 & $\begin{array}{l}0.69 \\
\end{array}$ & 0.73 & 0.07 & -0.08 & $\begin{array}{c}-0.49 \\
\end{array}$ & 0.22 & 0.25 & 0.18 & 0.00 & -0.28 & -0.57 & 0.02 & 0.46 & -0.37 & -0.34 & -0.89 & 1.00 \\
\hline
\end{tabular}

coordinates in the graph). Interpretation of the correlation between different parameters is based on the following rules: parameters located near each other are positively correlated, those separated by $180^{\circ}$ (diametrically opposed) are negatively correlated, and those separated by $90^{\circ}$ are noncorrelated. All parameters were found to be well represented in this $\mathrm{PC} 1 / \mathrm{PC} 2$ plot except $\mathrm{Ni}$ and $\mathrm{B}$ VisDy. SP and PEN are negatively and strongly correlated. SP showed a positive correlation with VisDy100, CCR, AspC5, AspC7, $\mathrm{V}, \mathrm{S}, \mathrm{Ha}$, and Har and a negative correlation with $\mathrm{C}, \mathrm{H}, \mathrm{H} / \mathrm{C}$, $\mathrm{N}$, and $\mathrm{Hb}$. Again, PEN shows also a similar correlation to these parameters but negative correlation becomes positive and vice versa. Many of the simulated distillation parameters have weak or no correlation with either SP or PEN. O and Hg parameters are also non-correlated to SP or PEN.

The results of correlations between different parameters and SP or PEN from the correlation matrix were very similar to those from PCA especially regarding the direction of the correlation (positive, negative, or non-correlated). From the correlation matrix and the PCA biplot, the following conclusions could be drawn: SP and PEN are inversely correlated to each other $[21,22]$. The increasing amount of asphaltenes in bitumen samples (increasing AspC5 and AspC7) is accompanied by an increase in SP and a decrease in PEN, similar to what was concluded by previous articles $[14,21,22]$. As the amount of asphaltenes increases, the viscosity of bitumen also increases (strong positive correlation). Bitumens with higher viscosity (higher VisDy100) are harder, this leads to decreasing PEN, and increasing SP. There is also a strong positive correlation between AspC5, AspC7 and CCR. Larger residue after combustion (increasing CCR) implies a harder bitumen with less PEN and higher SP. The following parameters are somewhat related to the aliphaticity/aromaticity of bitumen samples: $\mathrm{H}, \mathrm{H} / \mathrm{C}$ ratio, $\mathrm{Hg}, \mathrm{Hb}, \mathrm{Ha}$, and Har. Increasing aromaticity of bitumen samples (increasing $\mathrm{Ha}$ and Har - decreasing $\mathrm{H}, \mathrm{H} / \mathrm{C}$, and $\mathrm{Hb}$ ) is accompanied by increasing asphaltenes (the most aromatic fraction), viscosity, and consequently increasing SP, and decreasing PEN. It was found that B VisDy, O, N, or Ni content does not have a significant effect on either SP or PEN. On the other hand, bitumen samples with higher SP and lower PEN may have higher $\mathrm{V}$ and $\mathrm{S}$ content. A possible explanation is that the heavy metals and Sulphur usually concentrate in the asphaltenes fraction of bitumen $[2,3]$. It seems that simulated distillation (SD0.5\% to SD40\%) is generally not well correlated with SP or PEN. A possible cause might be the following: PEN and SP are significantly correlated with the heaviest fraction of the bitumen (asphaltenes and heavy resins) that cannot be vaporized even in a high temperature simulated distillation.

\subsection{Proposed models for prediction of SP or PEN}

The performance parameters of all the proposed models are summarized in Table 4. For each standard characterization test (SP or PEN), four models have been introduced. The first model is based on dynamic viscosity, the second one on FT-IR while the third one is based on H-NMR. The fourth model was built by using a combination of parameters. The performance parameters here include $R^{2}$, 


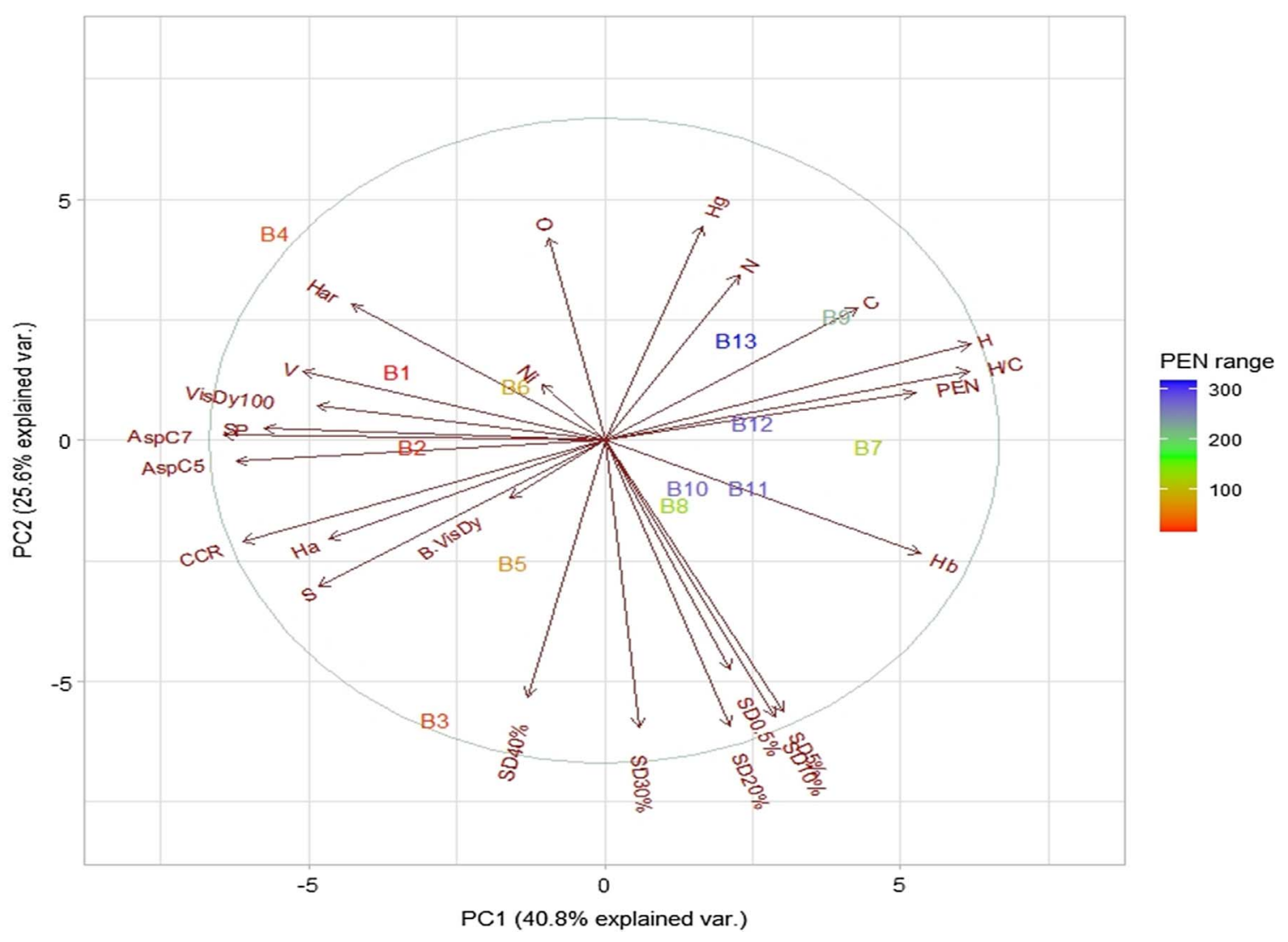

Fig. 1. Biplot of PC1 against PC2 for all parameters, which includes the scores (samples, numbered from B1 to B13) and loadings (the 25 parameters included in this study). The color grid refers to the PEN range of different samples.

RMSEC, RMSECV, and bias (for calibration and crossvalidation). The magnitude of $R^{2}$ describes the total variation in the predicted property that is explained by the model. The higher the $R^{2}$ value, the better is the model fit $\left(R^{2}\right.$ ranges from 0 to 1$)$. Two values are included: one for calibration and one for cross-validation. Mostly, the $R^{2}$ of the cross-validation will be less than that of the calibration. The closest the two $R^{2}$ value, the more robust the model. RMSEC indicates how much the estimated values of the calibration set agree with the actual values of this set. However, this parameter provides an optimistic estimate of the model ability of prediction as part of the noise in the calibration set is also modelled. Therefore, RMSECV serves as a better estimate and a more robust way of determining the prediction ability of the model as it is measured by applying the cross-validation techniques. Leave-One-Out (LOO) was the method of choice for crossvalidation in this study. This method is preferred when the size of the calibration set is small as in our case [25]. Both RMSEC and RMSECV were compared with the reproducibility of the standard methods of SP and PEN to determine how well each prediction model performs. Bias is an estimation of the systematic error. Low bias (near or equals zero) means that the model is accurate (the predicted values are very close to the measured values).

For the viscosity model of SP, the coefficients of determination $R^{2}$ for both calibration and cross-validation were high $(\geq 0.920)$ suggesting a good model which explains most of the total variation in SP. The RMSEC was equal to $2.2^{\circ} \mathrm{C}$ while it was $2.6^{\circ} \mathrm{C}$ for RMSECV. Both RMSEC and RMSECV were comparable to the reproducibility limit of the SP standard method $\left(2.0^{\circ} \mathrm{C}\right)$. Therefore, the proposed viscosity model has a very good prediction ability of SP. In addition, the bias of either calibration or crossvalidation was equal to zero, which means that the model is free from systematic error. As SP is taken as an approximate estimation of temperature at which the bitumen changes from a solid to a fluid (because bitumen has no definite melting point), it is not surprising that the SP has a strong correlation with the viscosity of bitumen (viscosity serves as a measure of the resistance of a fluid to flow) [2]. It can be concluded that SP of bitumen can be well predicted from dynamic viscosity at $100{ }^{\circ} \mathrm{C}$. The proposed model is presented in equation (2). Its form (logarithmic) is different from what was introduced by Oyekunle and Omatsone [20] (polynomial):

$$
\mathrm{SP}=9.2161 \times \ln (\mathrm{VisDy} 100)-26.111
$$

Compared to SP, the quality of the viscosity model of PEN, despite being one of the best PEN models, was significantly lower. The values of $R^{2}$ of calibration and cross-validation were relatively high $(\geq 0.930)$. However, the values of RMSEC and RMSECV were 25 and $31 \mathrm{dmm}$ respectively 
Table 4. Performance parameters of the different proposed models for prediction of SP or PEN.

\begin{tabular}{|c|c|c|c|c|c|c|c|c|c|}
\hline \multirow{2}{*}{$\begin{array}{l}\text { Predicted } \\
\text { Property }\end{array}$} & \multirow{2}{*}{$\begin{array}{l}\text { Variable(s) used in } \\
\text { Modelling }\end{array}$} & \multirow{2}{*}{$\begin{array}{l}\text { Type of } \\
\text { Model }\end{array}$} & \multirow{2}{*}{$\begin{array}{l}\text { Number of PCs } \\
\text { (For PLS models) }\end{array}$} & \multicolumn{3}{|c|}{ Calibration } & \multicolumn{3}{|c|}{ Validation (LOO) } \\
\hline & & & & $R^{2}$ & RMSEC & $\overline{\text { Bias }}$ & $R^{2}$ & RMSECV & $\overline{\text { Bias }}$ \\
\hline \multirow[t]{4}{*}{$\mathrm{SP}\left({ }^{\circ} \mathrm{C}\right)$} & VisDy100 & Log Model ${ }^{\mathrm{a}}$ & - & 0.976 & 2.2 & 0 & 0.920 & 2.6 & 0 \\
\hline & FT-IR & PLS & 8 & 0.999 & 0.5 & 0 & 0.977 & 2.3 & 0.5 \\
\hline & H-NMR & PLS & 2 & 0.320 & 11.7 & 0 & 0.055 & 14.0 & -1.7 \\
\hline & $\begin{array}{l}\text { VisDy100, AspC5, } \\
\text { AspC7, N, SD5\%, SD30\% }\end{array}$ & PLS & 4 & 0.995 & 1.0 & 0 & 0.987 & 1.7 & 0.2 \\
\hline \multirow[t]{4}{*}{ PEN (dmm) } & VisDy100 & Power Model & - & 0.936 & 25 & 0 & 0.930 & 31 & -7 \\
\hline & FT-IR & PLS & 5 & 0.912 & 35 & -1 & 0.717 & 65 & -2 \\
\hline & H-NMR & PLS & 2 & 0.206 & 102 & 0 & 0.026 & 117 & 8 \\
\hline & $\begin{array}{l}\text { VisDy100, CCR, } \\
\text { H/C, SD0.5\%, SD } 30 \%\end{array}$ & PLS & 4 & 0.988 & 13 & -2 & 0.973 & 20 & -6 \\
\hline
\end{tabular}

${ }^{\mathrm{a}}$ Log Model $=$ logarithmic model.

and both of them exceeded the reproducibility limit of the standard method (for PEN $<50,4 \mathrm{dmm}$ and for PEN $\geq 50,8 \%$ of the mean of the two results of PEN test). The error was higher in bitumen samples with higher penetration. The correlation between viscosity and PEN was moderate $(-0.64)$ while the correlation between viscosity and SP was strong (0.91) (Tab. 3), which can explain the lower performance of the viscosity model of PEN compared to that of SP. Nevertheless, the viscosity model of PEN can still serve as a tool for providing a preliminary estimation or a trend of PEN based on dynamic viscosity at $100{ }^{\circ} \mathrm{C}$. The viscosity model equation for PEN prediction is presented in equation (3):

$$
\mathrm{PEN}=26224 \times(\text { VisDy100 })-0.694
$$

Regarding the FT-IR model of SP (Fig. 2), SP was used as Log SP and the following preprocessing methods were applied to the FT-IR spectra: MSC (Mean), Baseline (Automatic Weighted Least Squares - 3rd order), and Smoothing (SavGol - 3rd order). The quality of this model was slightly better than the quality of the viscosity model. $R^{2}$ of calibration $=0.999$ and $R^{2}$ of cross-validation $=0.977$ indicate the significance of the description and prediction of SP using FT-IR. RMSEC and RMSECV were relatively lower compared to the viscosity model $\left(0.5\right.$ and $2.3{ }^{\circ} \mathrm{C}$ respectively). The proposed model has zero calibration bias while the cross-validation bias was around $0.5{ }^{\circ} \mathrm{C}$. By measuring either viscosity or FT-IR spectrum of a bitumen sample, an accurate prediction of SP of bitumen can be achieved that is comparable to the standard method. Besides, these methods are fast and cheap. Compared to the model proposed by Weigel and Stephan [11], this model is more accurate and covers a wider range of SP. Although the models proposed by Sun et al. [12] are relatively more accurate in theory, the samples used for modelling had a very narrow range of SP.

Log PEN was also used for the FT-IR model of PEN (Fig. 3). The following preprocessing was used: EMSC (Extended Scatter Correction) and Derivative
(SavGol - 1st order). The $R^{2}$ of cross-validation was low (0.717), while the RMSEC and RMSECV values were quite high (35 and $65 \mathrm{dmm}$ respectively). The FT-IR model of PEN had very low accuracy especially compared to the viscosity model. Such results might be attributed to the following observation: the prediction error was low in bitumen samples with lower PEN and it was very high in samples with higher penetration (Fig. 3). A more practical approach might be the developing of two different models: one for samples with low penetration and one for samples with higher penetration. This is on the contrary to what was suggested by Weigel and Stephan [11] and Sun et al. [12] but again, both of these articles had used bitumen samples with a very low and a very narrow range of PEN compared to this study and possibly with less diversity of the origins of these samples. Prediction of PEN using FT-IR is not recommended as the accuracy of prediction will be very low and further investigations are needed in order to assess the suggestion made before regarding the possibility of developing two models for PEN prediction from FT-IR data (one at lower PEN ranges and one at the higher ones).

An attempt was made to develop two models based on the H-NMR data using the four integration areas mentioned before: $\mathrm{Hg}, \mathrm{Hb}$, Ha, and Har. No preprocessing has been used as the four modelling parameters have the same units. Neither the SP model nor the PEN model showed even an acceptable accuracy. Values for $R^{2}$ for both models were very low $(\leq 0.320)$. RMSEC and RMSECV were also extremely higher than the reproducibility limit of the standard methods of SP and PEN (Tab. 4). The models developed by Jaimes et al. [10] involved the use of either LF-NMR alone or in combination with H-NMR data for the prediction of SP or PEN. The T2 relaxation time distribution measured by LF-NMR can indirectly reflect the composition of the bitumen samples through the impact of each component of the bitumen samples on the general molecular dynamics [10]. However, these proposed models were built using samples produced by one refinery and they belong to the same penetration grade. Such low diversity of the used samples makes it difficult to draw general 


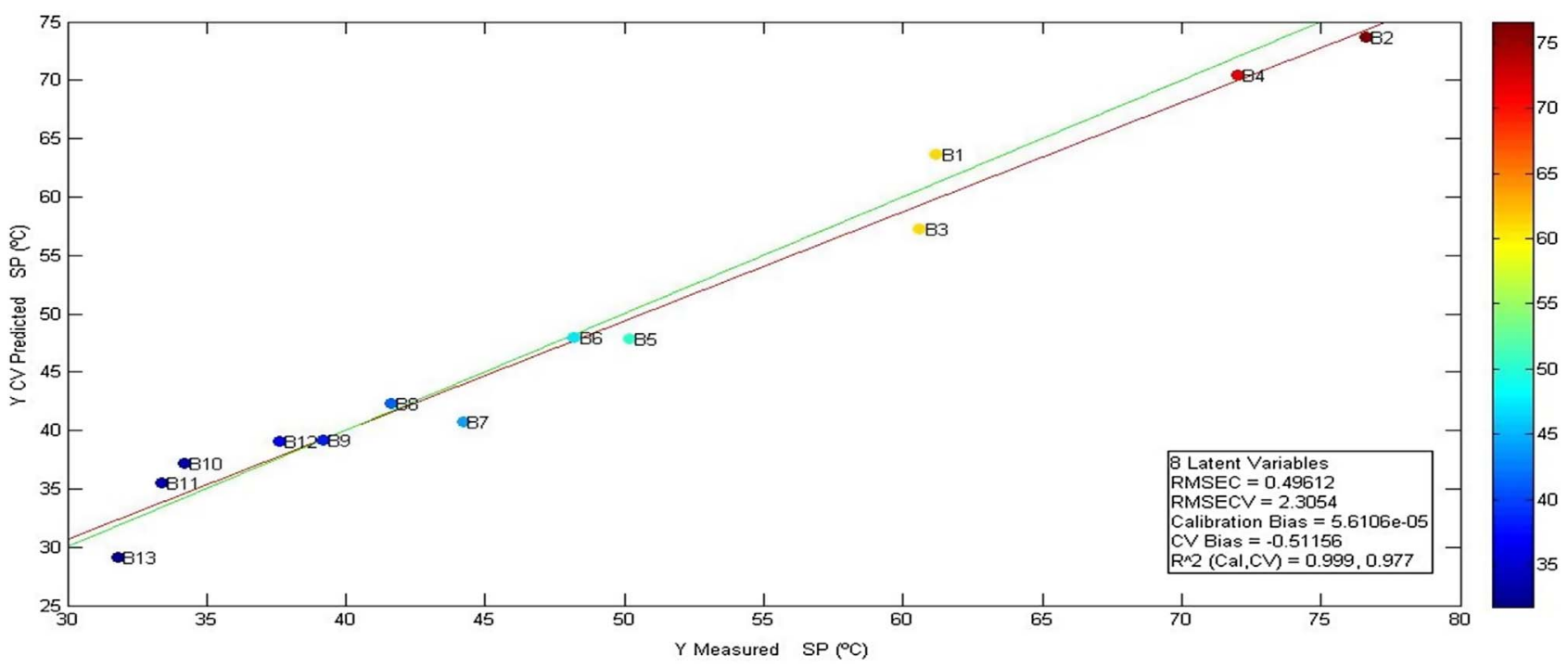

Fig. 2. Comparison between the measured values of SP and the calculated ones from cross-validation using FT-IR data (the green line is the perfect fit line while the red one is the model-fit line - samples are colored based on SP values - colors are represented on the color grid on the right).

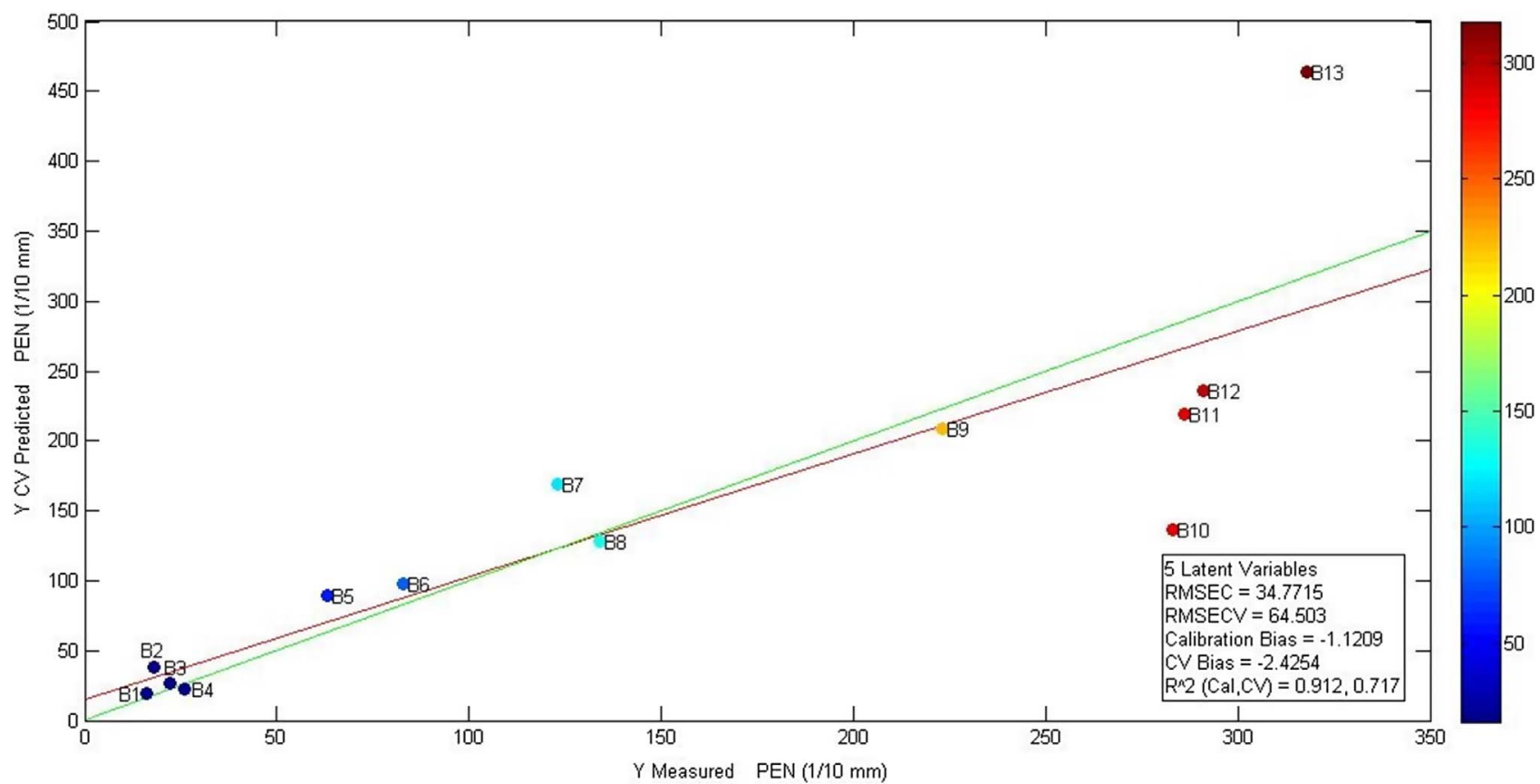

Fig. 3. Comparison between the measured values of PEN and the calculated ones from cross-validation using FT-IR data (the green line is the perfect fit line while the red one is the model-fit line - samples are colored based on PEN values - colors are represented on the color grid on the right).

conclusions regarding the suitability of either LF-NMR and/or H-NMR for the prediction of SP and PEN. Based on the results of our study, H-NMR cannot be used alone to model SP or PEN. By looking at the results obtained for the three prediction models of PEN (viscosity, FT-IR, and H-NMR models), it appears that the prediction of penetration is more challenging than the prediction of the softening point and no single property or technique can accurately describe and predict it.

Owing to the nature of SP and PEN tests (empirical tests), it is difficult to directly link any of them to a single property or a component of bitumen samples (especially PEN) but they rather depend on the whole composition of the bitumen samples and their molecular interactions 


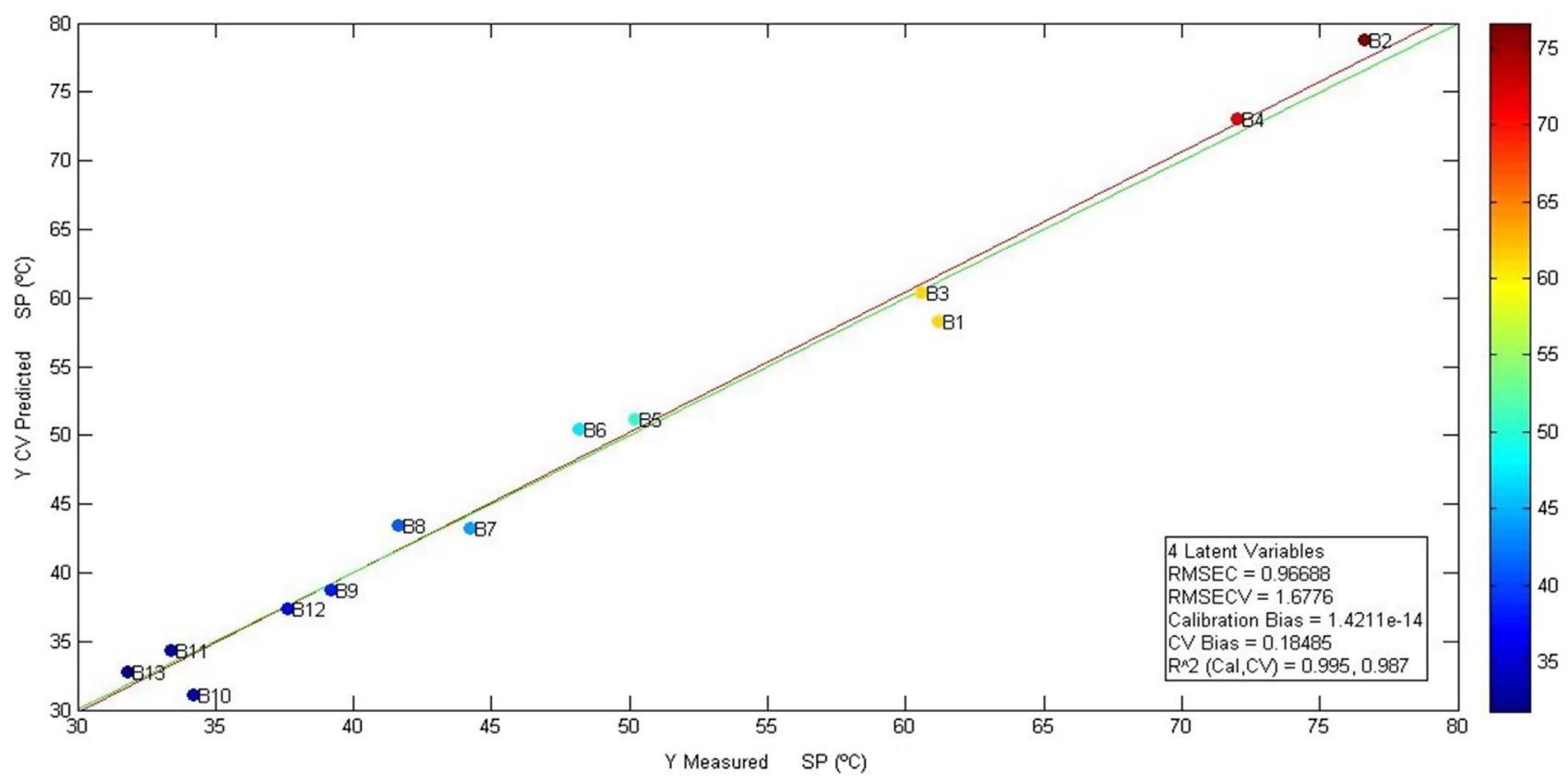

Fig. 4. Comparison between the measured values of SP and the calculated ones from cross-validation obtained from the multiparameters model (the green line is the perfect fit line while the red one is the model-fit line - samples are colored based on SP values colors are represented on the color grid on the right).

and dynamics [22]. Therefore, it might be possible to propose good models for the prediction of PEN and better models for the prediction of SP using a combination of parameters and analytical techniques. Many multiparameter models have been tested but only the best two multiparameter models will be discussed here: one for SP and one for PEN (Tab. 4). For the SP prediction, the multiparameter model showed a better accuracy compared to the other proposed models according to the values of the cross-validation parameters (Fig. 4). Six parameters were used for the developing of the best multiparameter model for SP prediction: dynamic viscosity at $100{ }^{\circ} \mathrm{C}$ (VisDy100), asphaltenes $\mathrm{C} 5$ content (AspC5), asphaltenes $\mathrm{C} 7$ content (AspC7), nitrogen content (N), temperatures at which 5\% and $30 \%$ of the weight of the original samples were "distillated" by GC Simulated Distillation (SD5\% and SD30\%). A remarkable observation about this model is that the use of nitrogen content in modelling was essential to obtain such an accurate model, although the correlation of nitrogen content with SP is not significant $(r=0.02$, Tab. 3$)$. All the parameters were autoscaled, including the SP. The coefficients of determination $R^{2}$ for both calibration and cross-validation were very high $(\geq 0.987)$, so it can be assumed that the multi-parameter model of SP can well describe most of its variation in bitumen samples. RMSEC was equal to $1.0^{\circ} \mathrm{C}$, which is a little bit higher than that of the FT-IR model. On the other hand, RMSECV of the multiparameter model was the lowest among the four proposed models $\left(1.7^{\circ} \mathrm{C}\right)$. Both RMSEC and RMSECV are lower than the reproducibility limit of the standard method. The bias of either calibration or cross-validation was also low $\left(<0.2{ }^{\circ} \mathrm{C}\right)$. As mentioned earlier, RMSECV is more robust in the assessment of the model accuracy when compared to RMSEC. Consequently, it can be concluded that the multiparameter model of SP is the best model among the four proposed models in terms of the accuracy of prediction.

Regarding the multiparameter model of PEN (Fig. 5), preprocessing of all parameters was done using logarithmic values of parameters (including PEN), followed by autoscaling. The following parameters were used in the model: dynamic viscosity at $100{ }^{\circ} \mathrm{C}$ (VisDy100), Conradson Carbon Residue (CCR), hydrogen to carbon ratio $(\mathrm{H} / \mathrm{C})$, temperatures at which $0.5 \%$ and $30 \%$ of the weight of the original samples were "distillated" by GC Simulated Distillation (SD0.5\% and SD30\%). All the previous parameters have a significant correlation with PEN except the simulated distillation parameters. The high $R^{2}$ values indicate the significance of the description and prediction of PEN by the multiparameter model. Moreover, the RMSEC (13 dmm) and RMSECV (20 dmm) were lower than that of the other proposed model for PEN prediction. Although the values of RMSEC and RMSECV are above the reproducibility limit of the standard method, this model can still be employed to give acceptable predictions of PEN compared to the other models.

In principle, the results of this section show the possibility of modelling SP or PEN using different physical and chemical properties of bitumen. Modelling of SP was quite easier compared to PEN with three out of the four proposed models (all except H-NMR model) showed the ability to provide an accurate prediction of SP comparable to the standard method. Conversely, the prediction of PEN was more demanding with no single property or technique (like viscosity or FT-IR spectra) has the ability to fully describe the variation of PEN in different samples. Only the 


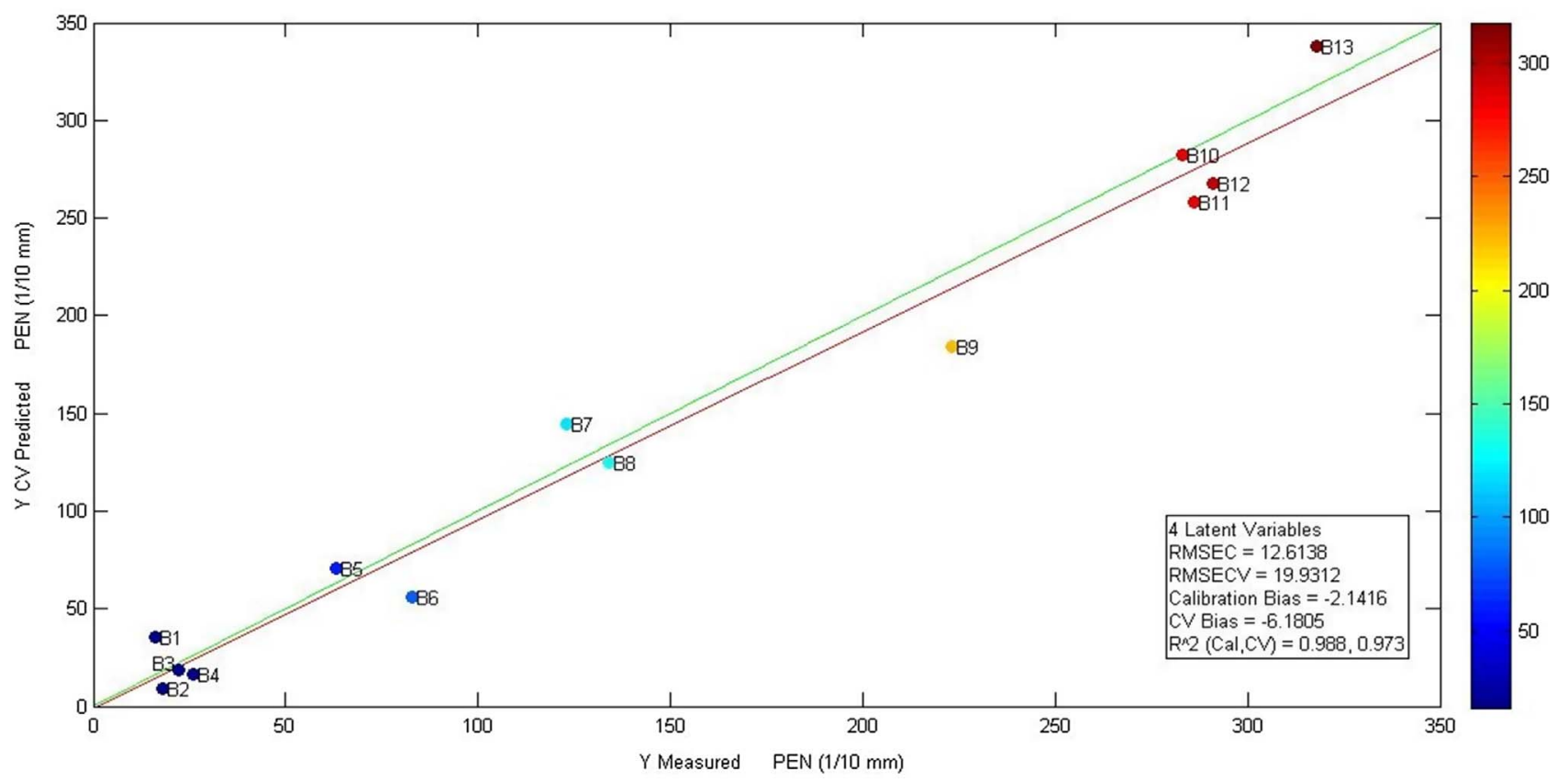

Fig. 5. Comparison between the measured values of PEN and the calculated ones from cross-validation obtained from the multiparameters model (The green line is the perfect fit line while the red one is the model-fit line - samples are colored based on PEN values - colors are represented on the color grid on the right).

multiparameter model showed an acceptable accuracy. Still, the viscosity model can be used to give a simple but very rough estimation of PEN. These findings support the previous assumption that the modelling of PEN may require a combination of parameters to obtain a reliable and accurate model.

Owing to the small sample size, it was not possible to make external validation of the proposed models. Such validation is important to ensure that the produced model is not overfitted especially for models with many parameters and low samples size. Therefore, it might be possible that some of the produced models are somewhat overfitted. To decrease such possibility, cross-validation was performed. Although the size of the samples set is small (13 samples), the diversity of these samples and the techniques used can serve in providing news insights and detecting trends regarding the relation of $\mathrm{SP}$ or $\mathrm{PEN}$ with the different used techniques and properties and the possibility of modelling these two tests using a combination of physical and chemical parameters.

\section{Conclusion}

Bitumen characterization remains a challenging task owing to its extreme complexity. More effort is still needed to achieve more knowledge of the relationship between the physical, chemical properties of bitumen and SP and PEN tests. In this study, characterization of 13 different bitumen samples was done using a variety of physicochemical properties and techniques. These multi-criteria analyses were studied to determine their correlation with two standard characterization tests of bitumen: Softening Point (SP) and Penetration (PEN) using correlation matrix and Principal Component Analysis (PCA). Some predictive models for SP or PEN tests have been proposed based on viscosity, FT-IR, H-NMR or a combination of parameters. The following conclusions could be drawn:

1. Both SP and PEN showed significant correlation with most of the analyses and parameters included except slope of dynamic viscosity (B VisDy), N, O, Hg, and many of simulated distillation but opposite in direction. Dynamic viscosity at $100^{\circ} \mathrm{C}$ (VisDy100), Conradson Carbon Residue (CCR), asphaltenes parameters (AspC5 and AspC7), and some parameters related to aromaticity $(\mathrm{H}, \mathrm{H} / \mathrm{C}$ ratio) showed higher correlation to either SP or PEN compared to the rest of the parameters. Asphaltenes content is crucial to understand and explain the results of these correlations.

2. Among the four proposed models of the SP, three of them showed very good accuracy that is comparable to the standard method and one showed very low accuracy and an exceptionally high error of prediction (H-NMR model). In terms of accuracy, the multiparameters model is the best one (root mean squared errors are below the reproducibility limit of the standard method). It shall be noticed the importance of introducing the nitrogen content to improve this model even if not directly correlated with the SP. However, using either dynamic viscosity or FT-IR is more convenient for the prediction of SP (simple and fast tests - no need to perform many analyses like in the multi-parameters model). 
3. Regarding the prediction of PEN, it appears that it is more challenging than SP prediction and no single property or parameter can well describe or predict the variation of PEN between different samples. Therefore, only the multi-parameters model showed an acceptable accuracy of prediction. The viscosity model can be used to give a very rough estimation of PEN.

4. Although the sample set used in this study is very diverse and covers a very wide range of SP or PEN, it is recommended that this work is reproduced by using a larger amount of samples, other types of bitumen samples (such as air-blown bitumens or bitumens with polymer additives), and more analytical techniques to further assess the correlation of many properties of bitumen with SP and PEN. External validation is needed in order to ensure that models are not overfitted, especially for the multiparameter models. The scope of this work can be extended in the future to study the correlation between these different analytical techniques and the evolution of SP or PEN with different standard aging tests like Rolling Thin film Oven Test (RTFOT) and Pressure Aging Vessel (PAV).

\section{References}

1 EN 12591:2009 (2009) Bitumen and bituminous binders Specifications for paving grade bitumens.

2 Sörensen A., Wichert B. (2012) Ullmann's Encyclopedia of Industrial Analysis: Asphalt and Bitumen, Wiley-VCH Verlag GmbH \& Co, KGaA, Weinheim.

3 Read J., Whiteoak D. (2003) The Shell Bitumen Handbook, 5 th edn., Thomas Telford, London.

4 Advantages of Asphalt: https://eapa.org/advantages-ofasphalt/ (accessed Oct. 1, 2020).

5 Nicholls J.C. (1998) Asphalt Surfacings - A guide to asphalt surfacings and treatments used for the surface course of road pavements, E \& FN SPON, London.

6 National Academies of Sciences, Engineering, and Medicine (2017) Relationship between chemical makeup of binders and engineering performance, The National Academies Press, Washington, DC.

7 Corbett L.W. (1969) Composition of asphalt based on generic fractionation, using solvent deasphaltening, elutionadsorption chromatography and densimetric characterization, Anal Chem. 41, 576-579.

8 Adams J.J., Elwardany M.D., Planche J.P., Boysen R.B., Rovani J.F. (2019) Diagnostic techniques for various asphalt refining and modification method, Energy Fuels 33, 2680-2698.

9 Nciri N., Kim J., Kim N., Cho N. (2016) An in-depth investigation into the physicochemical, thermal, microstructural, and rheological properties of petroleum and natural asphalts, Materials $\mathbf{9}, 859$.
10 Jaimes M.L., Santos S.N., Molina V.D. (2019) Prediction of the penetration grade and softening point of vacuum residues and asphalts by nuclear magnetic resonance and chemometric methods, Energy Fuels 33, 6264-6272.

11 Weigel S., Stephan D. (2017) The prediction of bitumen properties based on FTIR and multivariate analysis methods, Fuel 208, 655-661.

12 Sun X., Yuan H., Song C., Deng X., Lv G., Li X., Hu A. (2000) Rapid and simultaneous determination of physical and chemical properties of asphalt by ATR-FTIR spectroscopy combined with a novel calibration-free method, Constr. Build. Mater. 230, 116950.

13 Silva R.C., Radović J.R., Ahmed F., Ehrmann U., Brown M., Ortega L.C., Larter S., Pereira-Almao P., Oldenburg T.B.P. (2016) Characterization of acid-soluble oxidized asphaltenes by fourier transform ion cyclotron resonance mass spectrometry: insights on oxycracking processes and asphaltene structural features, Energy Fuels 30, 1, 171-179.

14 Weigel S., Stephan D. (2017) Modelling of rheological and ageing properties of bitumen based on its chemical structure, Mater. Struct. 50, 83.

15 Yoon S., Bhatt S.D., Lee W., Lee H.Y., Joeng S.Y., Baeg J., Lee C.W. (2009) Separation and characterization of bitumen from Athabasca oil sand, Korean J. Chem. Eng. 26, 64-71.

16 Tia M. (2013) Bituminous materials and mixtures civil engineering handbook, CRC Press Inc., Boca Raton, Florida.

17 EN 1427:2007 (2017) Bitumen and bituminous binders Determination of the softening point - Ring and Ball method.

18 EN 1426:2007 (2017) Bitumen and bituminous binders Determination of needle penetration.

19 Traxler R.N., Pittman C.U., Burns F.B. (1935) Rheological properties of Asphalt II, Discussion of Penetration Viscosity Relationships, Physics 6, 58.

20 Oyekunle L.O., Omatsone O.O. (2000) Mathematical modelling of the rheological properties of petroleum bitumens, Pet. Sci. 18, 5-6, 711-723.

21 Oyekunle L.O. (2006) Certain relationships between chemical composition and properties of petroleum asphalts from different origin, Oil Gas Sci. Technol. - Rev. IFP Energies nouvelles 61, 3, 433-441.

22 Oyekunle L.O. (2007) Influence of chemical composition on the physical characteristics of Paving Asphalts, Pet. Sci. Technol. 25, 11, 1401-1414.

23 Glen S. (2021) "Correlation coefficient: simple definition, formula, easy steps" from StatisticsHowTo.com: Elementary statistics for the rest of us! https://www.statisticshowto.com/ probability-and-statistics/correlation-coefficient-formula/.

24 Brereton R.G. (2003) Chemometrics: Data analysis for the laboratory and chemical plant, John Wiley \& Sons Ltd., West Sussex.

25 Gemperline P. (2006) Practical Guide to Chemometrics, Second Edition, CRC Press, Taylor \& Francis Group, London.

26 Krishnan M., Rajagopal K. (2003) Review of the uses and modeling of bitumen from ancient to modern times, Appl. Mech. Rev. 56, 2, 149-214. 